\title{
Establishment of microRNA, transcript and protein regulatory networks in Alport syndrome induced pluripotent stem cells
}

\author{
WENBIAO CHEN $^{1}$, DONGE TANG ${ }^{2}$, YONG DAI ${ }^{2}$ and HONGYAN DIAO ${ }^{1}$ \\ ${ }^{1}$ State Key Laboratory for Diagnosis and Treatment of Infectious Diseases, Collaborative Innovation Center for Diagnosis and \\ Treatment of Infectious Diseases, The First Affiliated Hospital, College of Medicine, Zhejiang University, \\ Hangzhou, Zhejiang 310003; ${ }^{2}$ Clinical Medical Research Center, The Second Clinical Medical College of Jinan University \\ (Shenzhen People's Hospital), Shenzhen, Guangdong 518020, P.R. China
}

Received January 29, 2018; Accepted July 12, 2018

DOI: $10.3892 / \mathrm{mmr} .2018 .9672$

\begin{abstract}
Alport syndrome (AS) is an inherited progressive disease caused by mutations in genes encoding for the $\alpha 3, \alpha 4$ and $\alpha 5$ chains, which are an essential component of type IV collagen and are required for formation of the glomerular basement membrane. However, the underlying etiology of AS remains largely unknown, and the aim of the present study was to examine the genetic mechanisms in AS. Induced pluripotent stem cells (iPSCs) were generated from renal tubular cells. The Illumina HiSeq ${ }^{\mathrm{TM}} 2000$ system and iTRAQ-coupled 2D liquid chromatography-tandem mass spectrometry were used to generate the sequences of microRNAs (miRNAs), transcripts and proteins from AS-iPSCs. Integration of miRNA, transcript and protein expression data was used to construct regulatory networks and identify specific miRNA targets amongst the transcripts and proteins. Relative quantitative proteomics using iTRAQ technology revealed 383 differentially abundant proteins, and high-throughput sequencing identified 155 differentially expressed miRNAs and 1,168 differentially expressed transcripts. Potential miRNA targets were predicted using miRanda, TargetScan and Pictar. All target proteins and transcripts were subjected to network analysis with miRNAs. Gene ontology analysis of the miRNAs and their targets revealed functional information
\end{abstract}

Correspondence to: Dr Yong Dai, Clinical Medical Research Center, The Second Clinical Medical College of Jinan University (Shenzhen People's Hospital), 1017 East Gate North Road, Shenzhen, Guangdong 518020, P.R. China

E-mail: daiyong22@aliyun.com

Dr Hongyan Diao, State Key Laboratory for Diagnosis and Treatment of Infectious Diseases, Collaborative Innovation Center for Diagnosis and Treatment of Infectious Diseases, The First Affiliated Hospital, College of Medicine, Zhejiang University, 79 Ching Chun Road, Hangzhou, Zhejiang 310003, P.R. China

E-mail: diaohy@zju.edu.cn

Key words: microRNA, transcript, protein, induced pluripotent stem cell, Alport syndrome on the iPSCs, including biological process and cell signaling. Kyoto Encyclopedia of Genes and Genomes pathways analysis revealed that the transcripts and proteins were primarily enriched in metabolic and cell adhesion molecule pathways. In addition, the network maps identified hsa-miRNA (miR)-4775 as a prominent miRNA that was associated with a number of targets. Similarly, the prominent ELV-like protein 1-A and epidermal growth factor receptor $(E G F R)$-associated transcripts were identified. Reverse transcription-quantitative polymerase chain reaction analysis was used to confirm the upregulation of hsa-miR-4775 and EGFR. The integrated approach used in the present study provided a comprehensive molecular characterization of AS. The results may also further understanding of the genetic pathogenesis of AS and facilitate the identification of candidate biomarkers for AS.

\section{Introduction}

Alport syndrome (AS) is a hereditary glomerulonephritis, which is variably associated with neural hearing loss and ocular abnormalities. It is widely accepted that AS is a genetically heterogeneous disease, which is caused by mutations in the collagen type IV $\alpha 5$ chain $(C O L 4 A 5)$ gene located at Xq22.3 and encoding the $\alpha 5$ chain of type IV collagen $(1,2)$. The primary pathological event appears to be the loss of the type IV collagen network, consisting of $\alpha 3, \alpha 4$ and $\alpha 5$ chains, which is an important component of the glomerular basement membrane (GBM). This results in the characteristic thinning, thickening and splitting of the $\operatorname{GBM}(3,4)$. Until now, a definitive diagnosis of AS was only possible through renal biopsy (5), which is primarily performed late following the onset of the profound clinical symptoms of this progressive renal disease. Therefore, early diagnosis and preemptive treatments have become increasingly important (6). Progress has been made in understanding the genetic basis of AS $(7,8)$ and improving treatment, although patients suffering from AS inevitably develop end-stage renal disease. A study demonstrated that the secretion of $\alpha 3-\alpha 4-\alpha 5$ (IV) heterotrimers by podocytes into a preformed, abnormal, filtering GBM is effective at restoring the missing collagen IV network, slowing kidney disease progression and extending life span (9). Another study identified a missense mutation, c.368G $>$ A (p.Gly123Glu), in 
the COL4A5 gene, which was reported to be the genetic cause of AS (10). However, the exact mechanisms remain poorly understood and novel treatment strategies are still required. Thus, novel analysis methods are required to reveal the genetic basis and mechanisms underlying AS.

MicroRNAs (miRNAs) regulate gene expression and modulate crucial biological processes, including differentiation, proliferation and apoptosis. They function through various mechanisms, including targeted miRNA degradation and translational repression $(11,12)$. Aberrant miRNA expression is associated with kidney disease. Emerging evidence from clinical and animal studies has indicated a critical role for miRNAs in renal pathophysiology (13). In previous studies, miRNAs were studied in the context of nephropathy, including membranous nephropathy, immunoglobulin (Ig) A nephropathy, lupus nephritis and renal transplantation. Collectively, the results demonstrated that miRNAs may act as biomarkers for the early diagnosis of kidney disease (14-16). Transcript sequencing is a useful alternative to whole genome sequencing as it avoids non-coding and repetitive sequences that make up the majority of eukaryotic genomes. Transcriptomic data offers an opportunity to deliver fast, inexpensive and accurate genome information (17). Transcriptome analysis of diabetic kidney disease identified multiple genes and pathways that may serve a role in the pathogenesis of the disease or act as biomarkers (18). A previous study demonstrated that injury-associated transcriptome alterations occur in kidney parenchymal cells in response to stresses of transplantation (19). Transcriptome analysis appears to be an effective and important tool for hypothesis-driven investigation of kidney disease. In addition, proteins are useful for gaining insight into the functional network of gene expression. With the advancement of 2D gel electrophoresis and isobaric tagging for relative and absolute quantification technologies, protein analysis has become an important tool for investigating disease (20). Exosomal proteomics has emerged as a powerful tool for understanding the molecular composition of exosomes and has the potential to accelerate biomarker discovery in diabetic nephropathy (21). By comparing Ig A nephropathy with a normal control, Park et al (22) established a urinary proteomic map of IgA nephropathy and identified 216 protein spots that were differentially expressed. Therefore, studying proteins may aid in understanding the molecular physiology of nephropathy and identifying early diagnostic markers.

Induced pluripotent stem cells (iPSCs) may be used to generate pluripotent patient-specific cell lines that are beneficial for studying the pathogenesis of model human diseases, and may also be used to determine genetic information associated with disease pathogenesis, particularly for diseases resulting from abnormal embryonic development $(23,24)$. Extensive research, including system-wide genomic analyses and comparisons of transcripts expression profiles, has been conducted to identify iPSCs (25). The molecular mechanisms that govern the induction, maintenance and directed differentiation of pluripotency are mediated by transcription factors and non-coding RNAs, including miRNAs and long non-coding RNAs $(26,27)$. Systematic deciphering of genetic or epigenetic alterations may help identify genomic hotspots in iPSCs with their consistent genetic background. However, these finding have also left notable gaps in the knowledge regarding the development and progression of disease.

In the present study, iPSCs from renal tubular cells of patients with AS (AS-iPSCs) and normal controls (NC-iPSCs) were successfully generated (28). Subsequently, miRNA, transcript and protein regulation in iPSCs was studied with the aim of better understanding the genetic mechanism of AS. Furthermore, the identified proteins, in addition to miRNAs and their target transcripts, were subjected to gene ontology (GO) and Kyoto Encyclopedia of Genes and Genomes (KEGG) functional analyses. The resulting comprehensive network maps of miRNAs, transcripts and proteins may help with understanding the pathogenesis of AS at the genetic level (Fig. 1).

\section{Materials and methods}

Patients and controls. A family with three generations of patients suffering from AS was identified (Fig. 2). The proband (III2) was a 26-year-old woman, who presented clinically with gross hematuria and albuminuria. The patient was diagnosed with AS at the Second Clinical Medical College of Jinan University (Shenzhen, China) in 2013. The proband was confirmed to have AS by pathological examination. The grandmother of the proband (I2) was also diagnosed with AS, although this individual succumbed to kidney failure. The mother of the proband (II3) exhibited AS symptoms, including kidney failure, gross hematuria, albuminuria, sensorineural hearing loss and pathognomonic ocular lesions. The sister of the proband (III3) presented with mild clinical symptoms of gross hematuria and albuminuria. The study protocol and consent forms were approved by the Ethics Committee of Jinan University and adhered to the guidelines set forth in the Declaration of Helsinki. Healthy participants and those with AS provided written informed consent.

A total of six members of the family were selected for further research. The proband (III2), her mother (II3) and a sister (III3) formed the experimental AS group, whereas a sister (III4), brother (III1) and her father (II4) served as the normal controls (NC) (Table I).

Renal tubular cell isolation and iPSC generation. iPSCs were successfully generated from renal tubular cells, as previously described (28). iPSC formation was confirmed by comparatively analyzing human embryonic stem cell (hESC) markers via colony morphology, immunohistochemistry, reverse transcription-quantitative polymerase chain reaction (RT-qPCR), flow cytometry, gene expression profiling of the three germ layers and karyotyping. The results demonstrated that iPSCs were similar to hESCs with regards to morphology, proliferation, hESC-specific surface marker expression and differentiation into the cell types of the three germ layers. Therefore, iPSCs have similar characteristics to hESCs and are suitable for AS research (28).

Total RNA from iPSCs was extracted using TRIzol ${ }^{\circledR}$ (Invitrogen; Thermo Fisher Scientific, Inc., Waltham, MA, USA), according to the manufacturer's protocol. RNA concentration was measured using a NanoDrop ${ }^{\mathrm{TM}} 2000$ spectrophotometer (Thermo Fisher Scientific, Inc.) and stored at $-80^{\circ} \mathrm{C}$. All materials and solutions were handled under RNase-free conditions, and all solutions were prepared with 


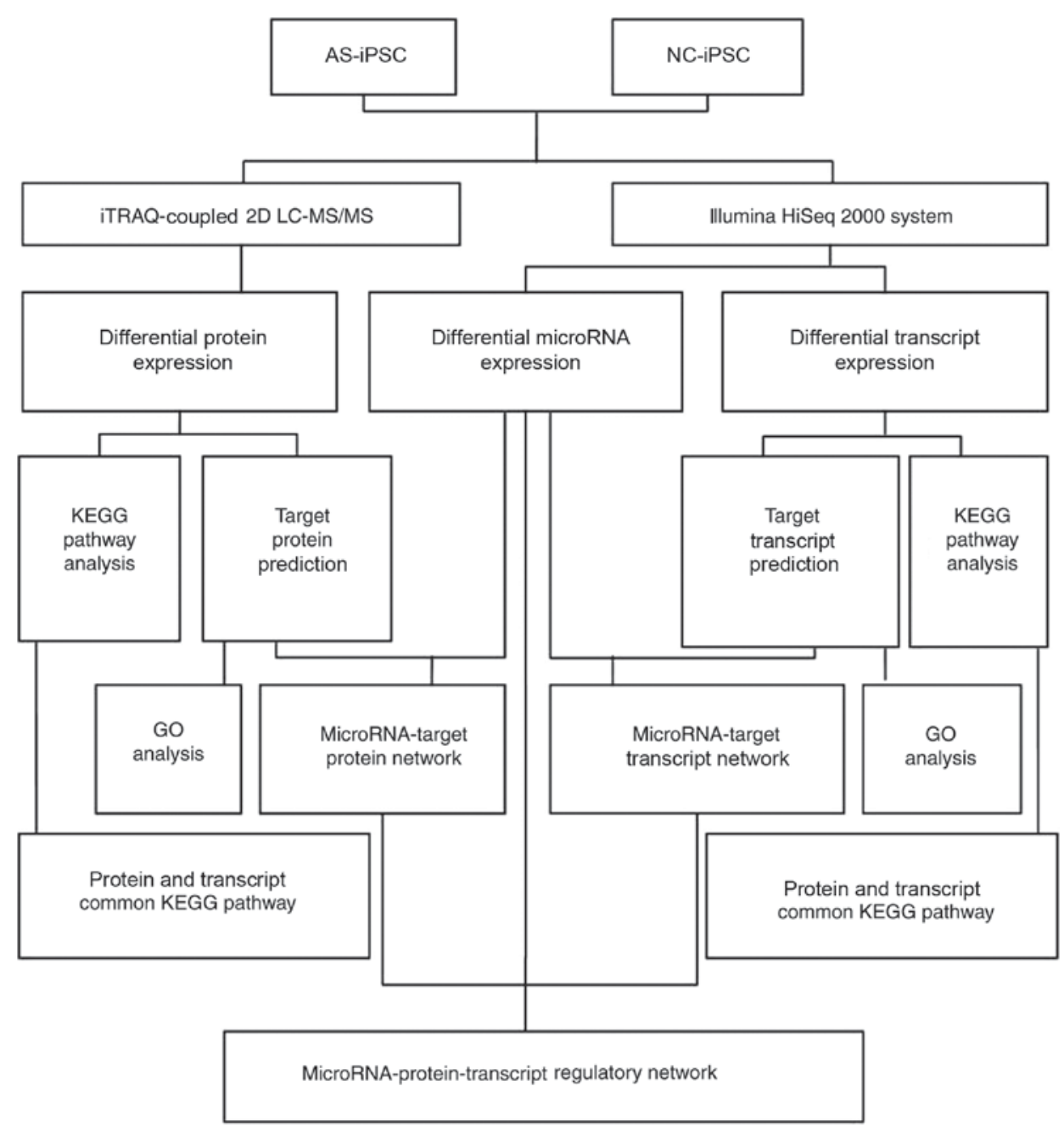

Figure 1. Schematic representation of the protocols of the present study. AS, Alport syndrome; GO, Gene Ontology; iPSC, induced pluripotent stem cell; KEGG, Kyoto Encyclopedia of Genes and Genomes; LC-MS/MS, liquid chromatography-tandem mass spectrometry; miRNA, microRNA; NC, normal control.

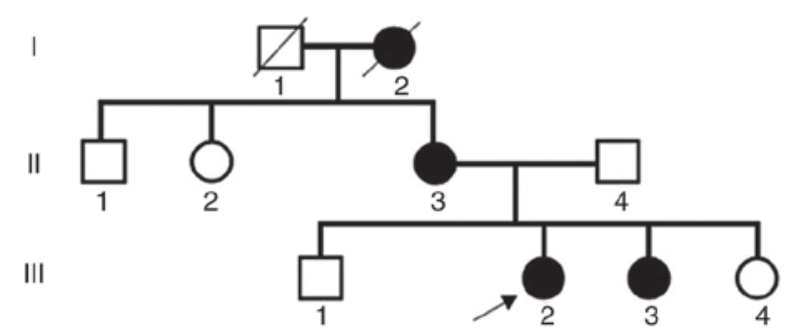

Figure 2. AS family pedigree chart. Males are represented by squares and females by circles. Black symbols indicate patients with AS. Slashes indicate deceased family members. The proband is indicated by the arrow. AS, Alport syndrome.

RNase-free water. Small RNAs (sRNAs) from the AS and NC groups were sequenced by BGI (Shenzhen, China) using Solexa high-throughput sequencing technology. A total of $\sim 2 \mu \mathrm{g}$ RNA/sample was used to prepare an sRNA library using the TruSeq sRNA sample preparation kit, according to the manufacturer's protocol (Illumina, Inc., San Diego, CA, USA). sRNA libraries were sequenced in two batches (three samples per sequencing run) using a next sequencing apparatus (Illumina HiSeq $^{\text {TM }}$ 2000), to generate $\sim 16$ million single-end 75 -bp reads per sample. To compare the estimated miRNA expression, the miRNA levels were first normalized. Subsequently, the fold change between $\mathrm{AS}$ and $\mathrm{NC}$ was calculated as follows: Fold change $=\log _{2}(\mathrm{AS} / \mathrm{NC})$. A rigorous significance test developed by Audic and Claverie (29) was used to identify differentially expressed genes with minimal statistical error. A fold change $\geq 1$ and $\mathrm{P} \leq 0.01$ were considered to indicate statistically significant differential expression between the two groups.

Following total RNA extraction, a cDNA library was constructed using the First Strand cDNA Synthesis kit (Thermo Fisher Scientific, Inc.) containing random oligonucleotides and a recombinant Moloney murine leukemia virus reverse transcriptase. The cDNA library consisted of 200-bp fragments. The cDNA whole transcriptome library was sequenced on the Illumina HiSeq ${ }^{\mathrm{TM}} 2000$ sequencing platform (Illumina, Inc.). Differentially expressed transcripts were detected by IDEG6 software (http://telethon.bio.unipd.it/bioinfo/IDEG6_form/) using a general $\chi^{2}$ test based on the reads per kilobase of transcript per million mapped reads (RPKM) values. Multiple testing correction via false discovery rate (FDR) was performed. An FDR $<0.001$, fold change $\geq 1$, and $\mathrm{P} \leq 0.05$ were used as thresholds to judge the significance of differential gene expression.

Proteome identification and comparative analysis. iPSCs were washed five times in ice-cold PBS and lysed using enhanced 
Table I. Participant characteristics and blood biochemical test results.

\begin{tabular}{llcccccccccc}
\hline Individual & Sex & $\begin{array}{c}\text { Age, } \\
\text { years }\end{array}$ & BLO & PRO & $\begin{array}{c}\text { Urea nitrogen, } \\
\mathrm{mmol} / \mathrm{l}\end{array}$ & $\begin{array}{c}\text { Inosine, } \\
\mu \mathrm{mol} / \mathrm{l}\end{array}$ & $\begin{array}{c}\mathrm{TP}, \mathrm{ALB} \\
\mathrm{g} / \mathrm{l}\end{array}$ & $\begin{array}{c}\mathrm{ALHOL}, \mathrm{l} \\
\mathrm{g} / \mathrm{l}\end{array}$ & $\begin{array}{c}\mathrm{CH} \text { mol/l } \\
\text { loss }\end{array}$ & $\begin{array}{c}\text { Vision } \\
\text { loss (L/R) }\end{array}$ \\
\hline III2 & Female & 26 & ++ & + & 6.6 & 121 & 57 & 35 & 5.76 & + & $0.2 / 1.0$ \\
III3 & Female & 23 & + & + & 3.9 & 110 & 71 & 45 & 4.45 & + & $0.4 / 1.0$ \\
III & Female & 51 & ++ & ++ & 3.5 & 231 & 70 & 43 & 4.68 & + & $0.2 / 0.8$ \\
II4 & Male & 53 & - & - & 3.0 & 55 & 75 & 52 & 3.81 & - & $1.0 / 1.0$ \\
III1 & Male & 21 & - & - & 3.2 & 56 & 66 & 45 & 4.61 & - & $1.5 / 1.0$ \\
III4 & Female & 17 & - & - & 3.2 & 50 & 75 & 43 & 4.98 & - & $1.2 / 1.1$ \\
\hline
\end{tabular}

The vision test was based on the Eggers visual chart. ALB, albumin; BLO, urine occult blood; CHOL, cholesterol; L, left; PRO, urine protein; $\mathrm{R}$, right; TP, total protein.

radioimmunoprecipitation assay buffer (Beyotime Institute of Biotechnology, Haimen, China) containing protease and phosphatase inhibitors for $30 \mathrm{~min}$ on ice. Subsequently, the samples were sonicated at $4^{\circ} \mathrm{C}$ for $10 \mathrm{~min}$ at $40,000 \mathrm{x}$ g, with ten cycles of 5 -sec bursts followed by 30 -sec cooling intervals. Cell debris was removed by centrifugation at $12,000 \mathrm{x} \mathrm{g}$ for $30 \mathrm{~min}$ at $4^{\circ} \mathrm{C}$ and the supernatants were collected. Protein concentration was measured using a bicinchoninic acid assay kit (Pierce; Thermo Fisher Scientific, Inc.). A total of $100 \mu \mathrm{g}$ protein per AS or NC sample was labeled with iTRAQ ${ }^{\circledR}$ reagents (Applied Biosystems; Thermo Fisher Scientific, Inc.). Proteins with $\geq 3$ peptides were considered valid for subsequent analysis. For each peptide, the ratio between the iTRAQ ${ }^{\circledR}$ label peak value and the sum of the intensities was calculated. The ratio was normalized to 1 and divided by the median of the ratio. Relative quantification of proteins was based on the ratio of the peak areas of the mass-to-charge ratio of 114 (AS) and 115 (NC) tandem mass spectrometry (MS/MS) spectra. Proteins with a fold change of $>1.2$ in peptide abundance ( $>1.2$ increased or $<0.83$ decreased) were considered to be significantly differentially abundant $(\mathrm{P}<0.05)$.

miRNA target prediction. Three different software packages were used to predict the miRNA target genes, including PicTar (http://pictar.mdc-berlin.de/), miRanda v5 (http://www.ebi. ac.uk/enright $\% 20 \mathrm{srv} / \mathrm{microcosm} / \mathrm{htdocs} /$ targets/v5/) and TargetScan Human 7.2 (http://www.targetscan.org/vert/). Genes identified by at least two of the three platforms were selected as ultimate target genes.

Data analysis and network visualization. For functional analysis of miRNAs, differentially abundant proteins, differentially expressed transcripts and miRNAs were combined with miRNA target predictions to form integrated regulatory networks. Statistical analyses were performed using R software (http://www.r-project.org/) with the appropriate KEGGSOAP packages (http://www.bioconductor.org/packages $/ 2.4 / \mathrm{bioc} / \mathrm{html} / \mathrm{KEGGSOAP.html).} \mathrm{The} \mathrm{network} \mathrm{was}$ visualized using Cytoscape 3.0 software (http://cytoscape. org).

Kyoto Encyclopedia of Genes and Genomes (KEGG) pathway analysis. KEGG pathways of the differentially expressed transcripts and differentially abundant proteins were annotated using the Database for Annotation, Visualization and Integrated Discovery gene annotation tool 6.8 (http://david.abcc.ncifcrf.gov). KEGG pathways were defined as significantly enriched in target candidates at a corrected $\mathrm{P} \leq 0.01$. This analysis tool was also able to predict the principal biological functions and pathways in which the candidate targets were involved.

Gene Ontology (GO) analysis. Target transcripts, proteins and miRNAs were subjected to GO analysis. The whole GO database was used as the background network to calculate the number of genes in each node. The hypergeometric distribution was used to test the enrichment of genes in each GO node. GO terms were classified as biological process, cellular component and molecular function, whereby $\mathrm{P}<0.05$ was considered statistically significant.

Validation using $R T-q P C R$. RT-qPCR was used to validate the results of deep sequencing analysis for miRNAs and transcripts. U6 and GAPDH were selected as the internal controls for miRNAs and transcripts, respectively. A total of $2 \mu \mathrm{g}$ total RNA from each sample was extracted using a Qiagen RNeasy Mini Extraction kit and reverse transcribed to cDNA using miScript Reverse Transcription kit (both from Qiagen GmbH, Hilden, Germany) according to the manufacturer's protocol. Gene-specific primers were listed in Table II. The reverse transcription products were amplified using the following PCR program with SYBR green reagent (Roche Diagnostics, Basel, Switzerland) on a ABI QuantStudio ${ }^{\mathrm{TM}}$ 6 Flex Real-Time PCR system (Thermo Fisher Scientific, Inc.): Polymerase activation at $95^{\circ} \mathrm{C}$ for $2 \mathrm{~min}$, followed by 40 cycles of $94^{\circ} \mathrm{C}$ for $10 \mathrm{sec}, 59^{\circ} \mathrm{C}$ for $10 \mathrm{sec}$ and $72^{\circ} \mathrm{C}$ for $40 \mathrm{sec}$. All reactions were run in triplicate. miRNA and transcript expression levels were normalized to the reference genes. Fold changes were determined and the relative quantification of gene expression data was performed using the $2^{-\Delta \Delta \mathrm{Cq}}$ method (30).

\section{Results}

Sequence profiling of miRNAs, transcripts and proteins. To build an integrated network of miRNAs, transcripts and proteins for AS-iPSCs, miRNA, transcript and protein expression level data for AS-iPSCs and NC-iPSCs were compared. 
Table II. RT-qPCR primer used in the validation assays.

\begin{tabular}{|c|c|c|}
\hline Primer name & Primer sequence $\left(5^{\prime} \rightarrow 3^{\prime}\right)$ & $\operatorname{Tm}$ \\
\hline $\operatorname{mir} 4461-\mathrm{R}$ & GTCGTATCCAGTGCGTGTCGTGGAGTCGGCAATTGCACTGGATACGACTATGGC & \\
\hline $\operatorname{mir} 4461-\mathrm{F}$ & TATGTACGTAGTCTAGGCC & 55 \\
\hline mir-4775-R & GTCGTATCCAGTGCGTGTCGTGGAGTCGGCAATTGCACTGGATACGACACCAAC & \\
\hline mir-4775-F & AAGCATTCTTTCATTGGTTGG & 55 \\
\hline mir-4651-R & GTCGTATCCAGTGCGTGTCGTGGAGTCGGCAATTGCACTGGATACGACCCCACC & \\
\hline $\operatorname{mir}-4651-\mathrm{F}$ & CGGCGACGGCGGGGT & 55 \\
\hline U6-R & GTCGTATCCAGTGCGTGTCGTGGAGTCGGCAATTGCACTGGATACGACAAAATATG & \\
\hline U6-F & GATTAGCATGGCCCCTGC & 55 \\
\hline$E G F R-F$ & AAAGTGGCCGCCATTTTAGA & 57 \\
\hline$E G F R-\mathrm{R}$ & CAACAATCACGCAAAGCTCC & \\
\hline$C X 3 C L 1-\mathrm{F}$ & CGTGCAGCAAGATGACATCA & 59 \\
\hline CX3CL1-R & TCCTTGACCCATTGCTCCTT & \\
\hline LRRC55-F & AATGGACACCCGAAACCTCA & 57 \\
\hline LRRC55-R & TGGCACATGGCTGAAATTGT & \\
\hline$F A M 18 B 1-\mathrm{F}$ & AATGGTTGGCCTACGTTGGT & 59 \\
\hline$F A M 18 B 1-\mathrm{R}$ & TGGACAGGCAATAAGTCCCA & \\
\hline$A U R K C-\mathrm{F}$ & AGCGCACAGCCACGATAATA & 59 \\
\hline$A U R K C-\mathrm{R}$ & TGCACAGACCAGCCAAAATC & \\
\hline RPS4Y1-F & ATGGCAAGGTTCGAGTGGAT & 59 \\
\hline RPS4YI-R & GATGCGGTGAACAGCAAAAC & \\
\hline GAPDH-F & ACCACAGTCCATGCCATCAC & 59 \\
\hline GAPDH-R & TCCACCACCCTGTTGCTGTA & \\
\hline
\end{tabular}

Tm, primer melting temperature; F, forward; R, reverse.

The comparison of AS-iPSC and NC-iPSC sRNA libraries revealed 830 differentially expressed miRNAs, of which 155 were significantly differentially expressed, including 79 upregulated and 76 downregulated miRNAs (data not shown). The miRNA sequences were submitted to the National Center for Biotechnology Information (NCBI) and assigned the accession no. SRP041435.

Following quality control and filtering, 26,021,874 and 27,551,343 clean reads were obtained from the AS-iPSC and NC-iPSC transcript libraries, respectively. Following application of the thresholds for significance, 1,168 significantly differentially expressed genes were identified, including 786 that were upregulated and 382 that were downregulated (data not shown). The raw transcript sequence data were deposited at the NCBI under the accession no. SRP041474.

For the proteomic analysis, a total of 15,553 iTRAQ-labeled peptides that mapped to a total of 3,431 proteins were identified and quantified. Among them, 899 proteins were predicted as differentially abundant between the AS-iPSC and NC-iPSC samples. Of these, 383 were significantly differentially abundant proteins (data not shown), including 227 upregulated and 156 downregulated proteins.

miRNA-target transcript regulatory network. To establish the miRNA-target transcript network, a systematic investigation was conducted using miRanda, TargetScan and Pictar to predict potential miRNA target transcripts. A transcript was classed as a potential target if two out of three platforms identified it as a miRNA target. The network map, which consisted of miRNAs and their target transcripts, was constructed using Cytoscape (Fig. 3). The results indicated that 156 miRNAs were associated with 382 target transcripts. It was observed that miRNAs and their target transcripts were mutually cross-regulated. Among them, the most prominent miRNA was hsa-miRNA (miR)-4775, which was associated with 27 transcripts.

To evaluate the functions of the target transcripts and miRNAs, GO functional enrichment analysis was conducted. A total of three ontologies, including molecular function (MF), cellular component (CC) and biological process (BP) were enriched by features of the transcript and miRNA datasets. In total, miRNAs were mainly involved in $9 \mathrm{BP}, 7 \mathrm{CC}$ and $4 \mathrm{MF}$ terms (Fig. 4A), and the target transcripts were mainly involved in five BP, 5 CC and five MF terms (Fig. 4B). Notably, certain terms involving miRNAs were also associated with the target transcripts. BP terms included 'cellular process' and 'biological regulation'. CC terms included 'cell physiology' and 'membrane part'. MF terms included 'cell activity' and 'cellular molecular binding'.

miRNA-target protein regulatory network. GO enrichment analysis of the miRNAs and target proteins was performed. Target proteins were mainly enriched in five BP, five CC and five MF terms (Fig. 4C). Compared with the miRNA GO enrichment analysis, certain GO terms for the target proteins were the same or similar to those of the miRNAs. 


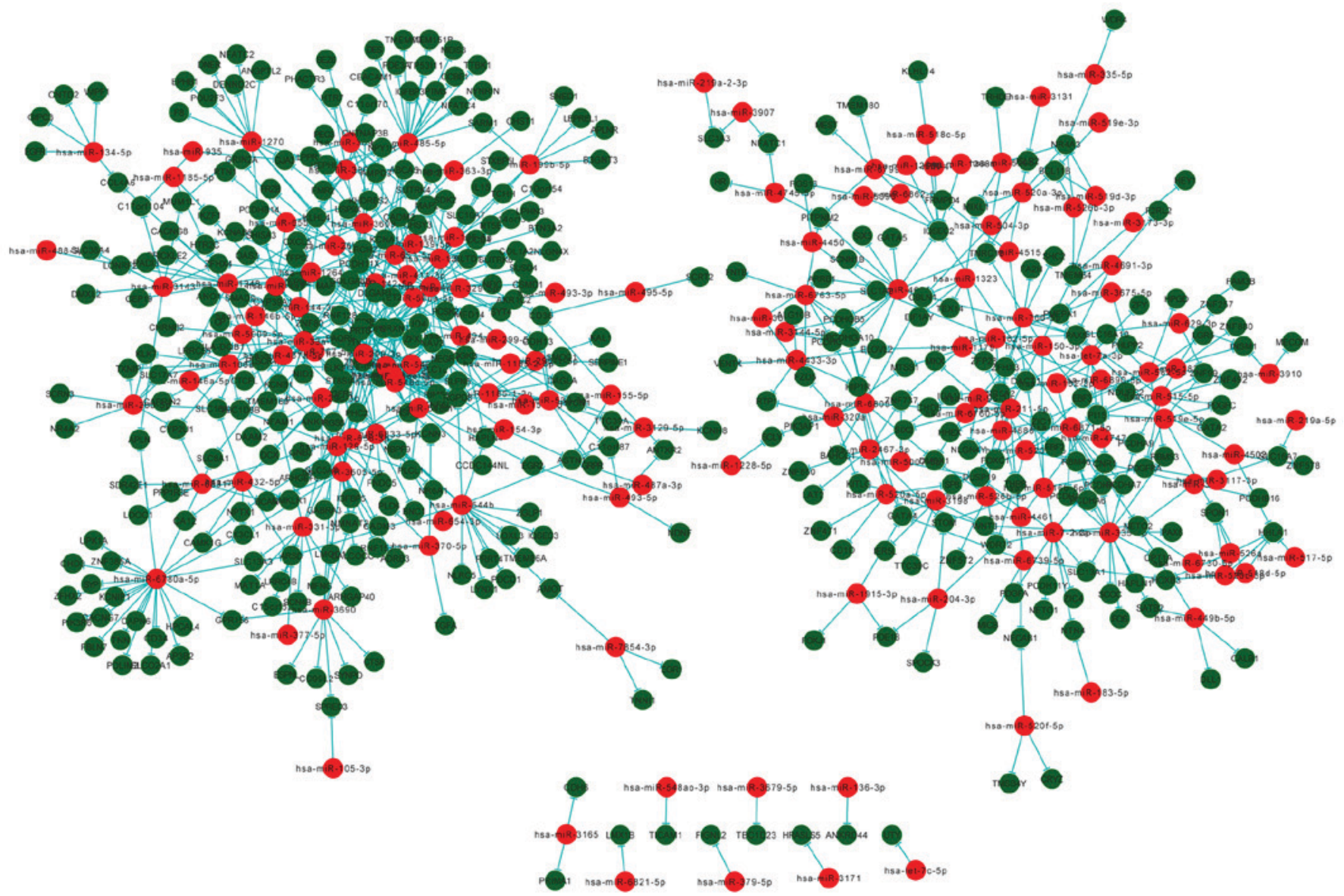

Figure 3. miRNA-target transcript regulatory network. A total of 156 miRNAs were associated with 382 target transcripts. Red circles represent upregulated miRNAs and green circles represent downregulated target transcripts. The lines signify coherent miRNA-target transcript pairs. miRNA, microRNA.

For example, BP included 'cellular physiological process' and 'intracellular signal', and MF included 'cellular activity' and 'cellular molecular binding'. However, the CC terms of the target proteins and miRNAs were different. The CC term 'intracellular biological process' was associated with the target proteins, whereas 'extracellular biological process' was associated with the miRNAs.

To investigate the potential functional association between miRNAs and target proteins, the differentially expressed miRNAs and target proteins were used to build a regulatory network. Cytoscape used to visualize the miRNA-target protein regulatory network and the results demonstrated that 26 miRNAs were associated with 25 target proteins (Fig. 5). In addition, the results indicated that the miRNAs and target proteins were mutually cross-regulated.

Transcript-protein regulatory network. A total of 1,168 differentially expressed transcripts and 383 differentially abundant proteins were used to build a regulatory network. The results demonstrated that 237 transcripts were associated with 189 proteins (Fig. 6). ELAV-like RNA binding protein 1 (ELAVL1) was the most prominent protein, which was associated with 41 transcripts, and epidermal growth factor receptor (EGFR) was the most prominent transcript, which was associated with 19 proteins.

KEGG was used to analyze pathways enriched for significantly differentially expressed transcripts between the
AS-iPSC and NC-iPSC libraries. Among them, 1,168 differentially expressed genes were involved in 15 pathways (data not shown) and 383 differentially abundant proteins were involved in 11 pathways (data not shown). Notably, there were several pathways that the proteins were involved in that were similar to those of the transcripts, including cell signaling pathways, cell metabolism pathways and cell interaction pathways. Transcripts and proteins were also mapped to KEGG pathways using GenMAPP to identify common pathways. The results indicated that the transcripts and proteins were primarily enriched in 262 common pathways, which included 'carbon metabolism' and 'cell adhesion molecules' (data not shown).

Numerous studies have demonstrated a substantial role for post-transcriptional, post-translational and protein degradation regulation in cellular biology (31). However, it is generally accepted to use transcript concentrations as proxies for the concentrations and activities of the corresponding proteins. On the basis of transcript-protein association, nine proteins and nine transcripts were identified to have a consistent expression pattern, including three upregulated and six downregulated pairs (Fig. 7).

miRNA-protein-transcript network. To integrate the miRNA, transcript and protein data, Cytoscape was used to construct a network comprising 156 miRNAs, 26 proteins and 381 transcripts (Fig. 8). The network consisted of miRNA-protein and miRNA-transcript interaction pairs, whereby a single miRNA 

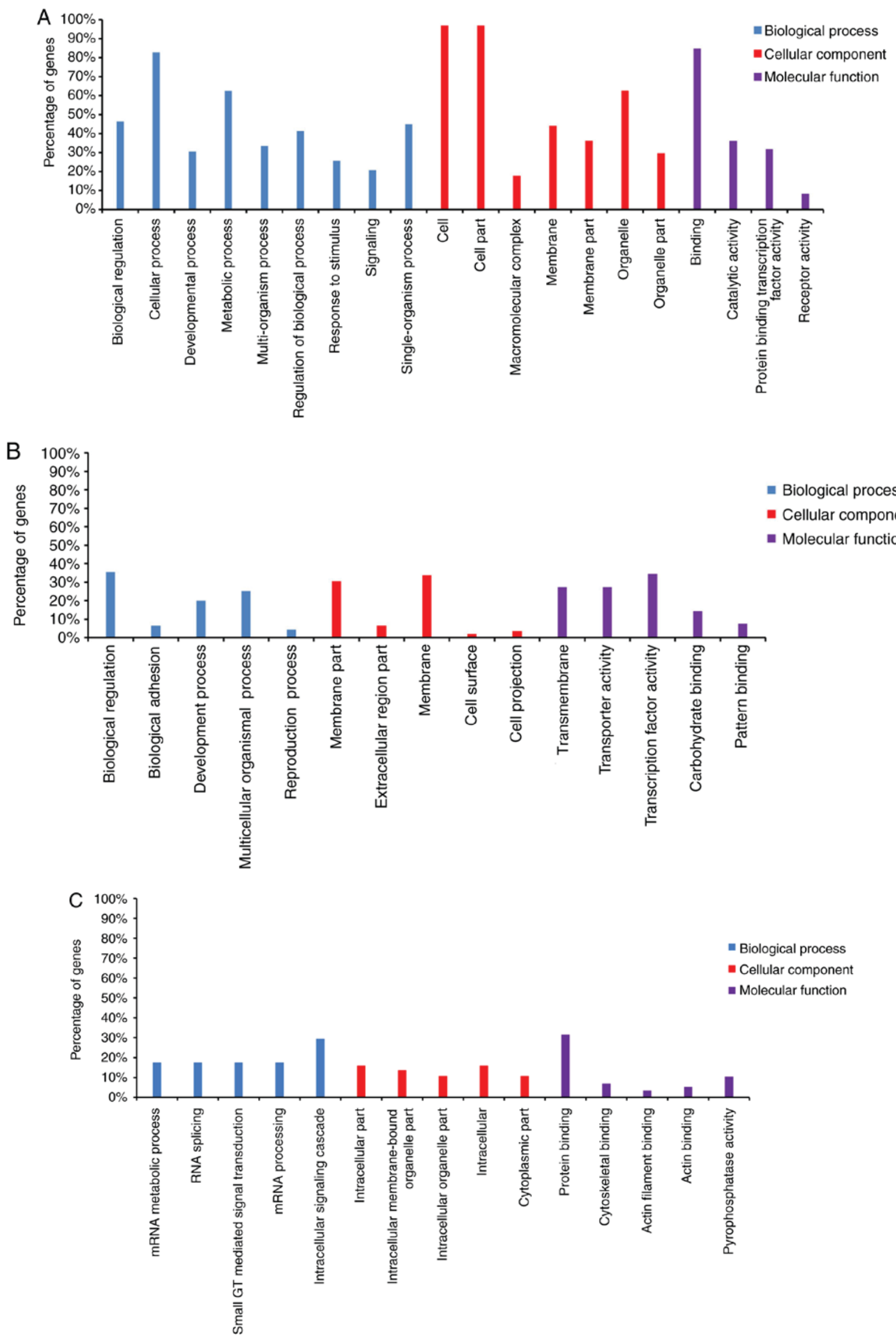

Figure 4. Enrichment of GO terms for miRNAs, target transcripts and target proteins. GO enrichments of (A) miRNAs, (B) target transcripts and (C) target proteins. The $\mathrm{X}$-axis indicates the GO ontology terms according to molecular function, cellular component and biological process. The Y-axis indicates the percentage of genes in each category. GO, gene ontology; miRNA, microRNA. 


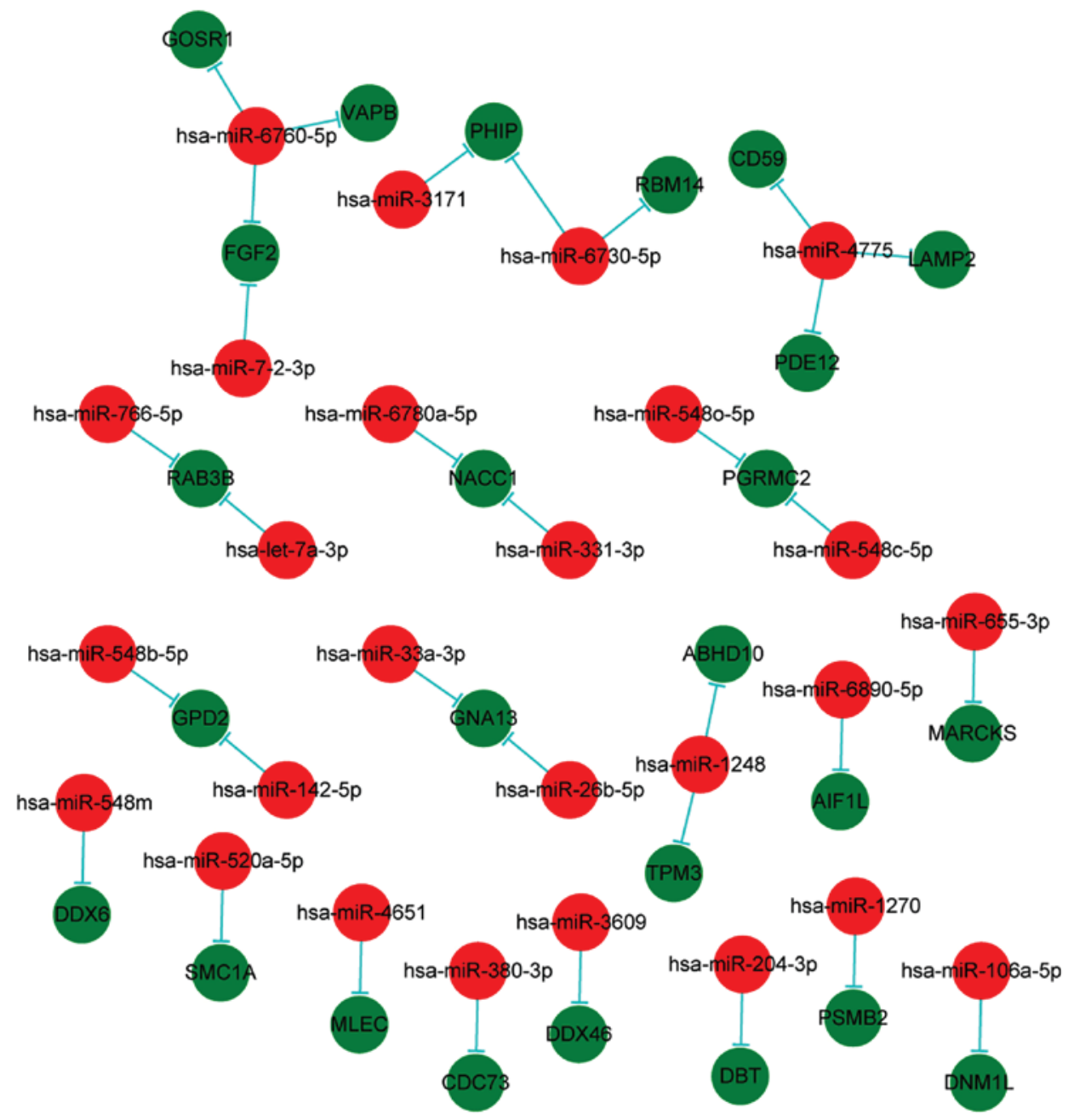

Figure 5. miRNA-target protein regulatory network. A total of 26 miRNAs were associated with 25 target proteins. Red circles represent upregulated miRNAs and green circles represent downregulated target proteins. The lines signify coherent miRNA-target protein pairs. miRNA/miR, microRNA.

was able to be connected to multiple transcripts or proteins, and a single protein or transcript was able to be targeted by multiple miRNAs. In the network, the most prominent miRNA was hsa-miR-4775, which was associated with 30 transcripts and proteins.

Expression profiling by $R T-q P C R$. A total of three differentially expressed miRNAs and six differentially expressed transcripts were selected for RT-qPCR analysis. These miRNAs and transcripts exhibited high abundance and were differentially expressed between the AS and NC groups. The expression levels determined by RT-qPCR were consistent with the read abundance of deep sequencing, indicating the validity of the microarray-based miRNA and transcript quantification. RT-qPCR confirmed that hsa-mir-4651, hsa-mir-4461 and hsa-miR-4775 were upregulated in AS compared with NC (data not shown). In addition, EGFR, LRRC55, and CX3CLI were upregulated; and AURKC, RPS4YI, and FAM18BI were downregulated in AS, compared with NC (data not shown).

\section{Discussion}

It is considered that IPSCs may revolutionize stem cell biology and regenerative medicine research by providing unprecedented opportunities to research human diseases and to overcome study limitations associated with safety, efficiency and ethics (32). IPSCs are an ideal platform for medical research as they carry the identical genetic anomalies associated with genetically heritable diseases, including Down's syndrome and type 1 diabetes. Various diseases have been modeled using iPSCs to better understand their etiology, which may aid in the development of novel treatments (33-35). In addition, iPSC technology has been employed in various diseases for disease modeling and gene therapy (36). Zou et al (37) demonstrated that single nucleotide substitution in human iPSCs was feasible, which may provide a novel strategy for gene therapy for monogenic diseases, including sickle cell disease. Another study used zinc finger nucleases and PiggyBac technology in human iPSCs to achieve biallelic correction of a point mutation (Glu342Lys) in the serpin family A member 1 gene, which is responsible for $\alpha 1$-antitrypsin deficiency (38). In addition, a previous study demonstrated successful in situ gene corrections in iPSCs derived from patients with pyruvate kinase deficiency (39). In the present study, iPSCs were used to study the pathogenic mechanisms of AS, since it is a hereditary disease caused by genetic mutations.

Increasing research has revealed that miRNAs, transcripts and proteins regulate iPSCs, which may be important for their 


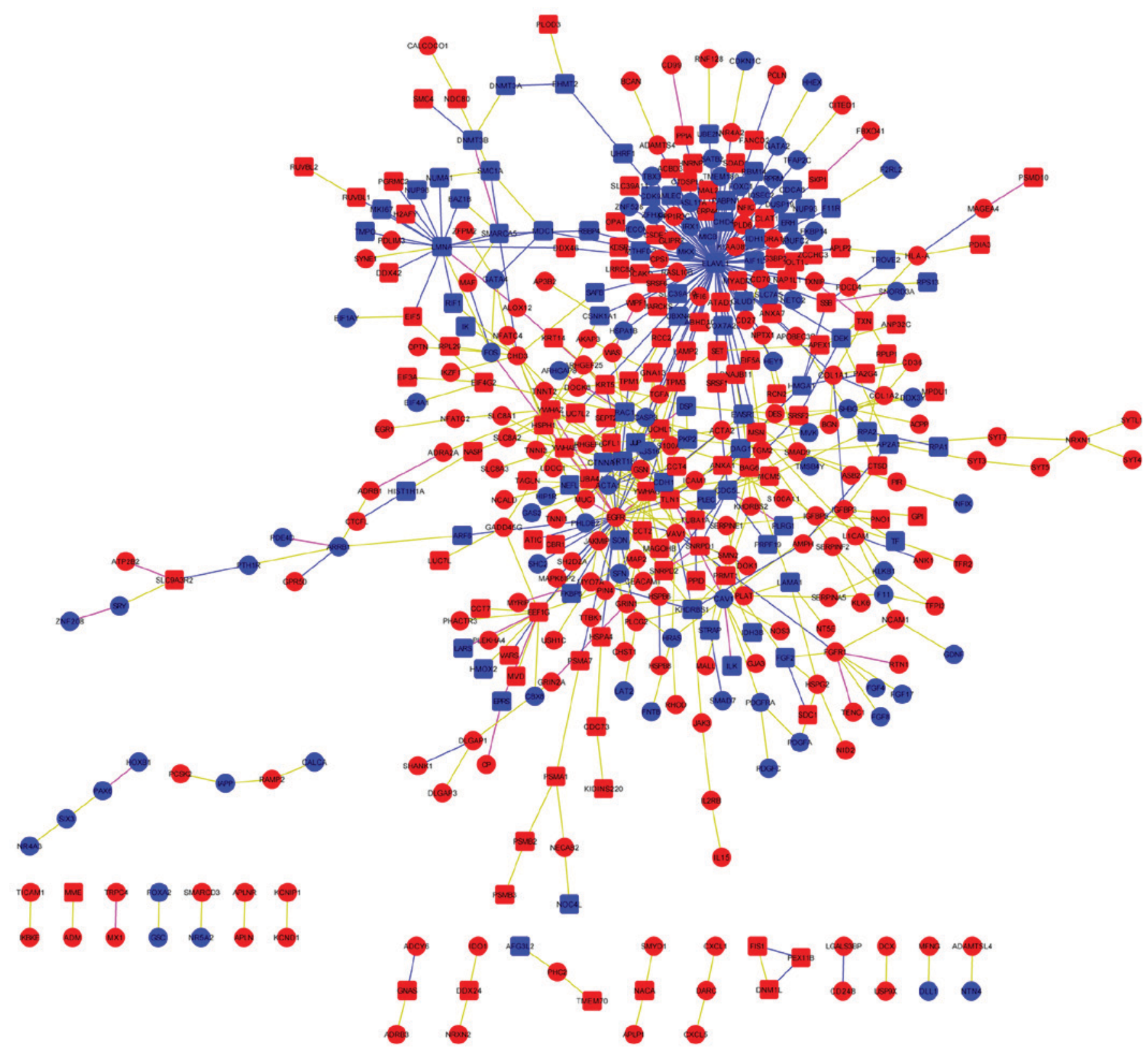

Figure 6. Transcript-protein regulatory network. A total of 237 transcripts were associated with 189 proteins. Circles represent transcripts and squares represent proteins. Red indicates upregulation and blue indicates downregulation. The lines signify coherent transcript-protein pairs.

pluripotency $(40,41)$. The specific regulatory roles of miRNAs in controlling the self-renewal and pluripotency of iPSCs are becoming increasingly evident. Studies have suggested a critical role for miRNAs in reprogramming somatic cells into pluripotent cells $(42,43)$. Another study revealed that cells undergoing iPSC reprogramming exhibited marked transcriptomic alterations associated with cell signaling pathways (44). Protein studies of iPSCs have also proven to have the potential to identify molecules and pathways that are important for iPSC biology $(45,46)$. Therefore, the present study made use of miRNA, transcript and protein data to study AS in an iPSC disease model, which will aid in a better understanding of AS pathogenesis.

Systems biological approaches provide tools to investigate the interactions between candidate genes by integrating miRNA, transcript and protein data. Network-based approaches are ideally suited to study the implications of functional genes in disease (47). In the present study, multiple expression profiling and bioinformatics analyses were integrated. The constructed databases were subjected to network analysis. To the best of our knowledge, the present study is the first of its kind to attempt investigating miRNA-target transcript, miRNA-target protein and transcript-protein networks in the context of an iPSC AS disease model. The results may be used for further research in the area of AS.

In the present study, significantly differentially expressed miRNAs, transcripts and proteins were identified, and the corresponding targets of the miRNAs were predicted. Target transcripts, proteins and miRNAs were subjected to GO enrichment analysis. A systematic comparison of the results revealed similar GO enrichments terms for AS-iPSCs and $\mathrm{NC}$-iPSCs. Cellular process in BP, membrane part in $\mathrm{CC}$, and 


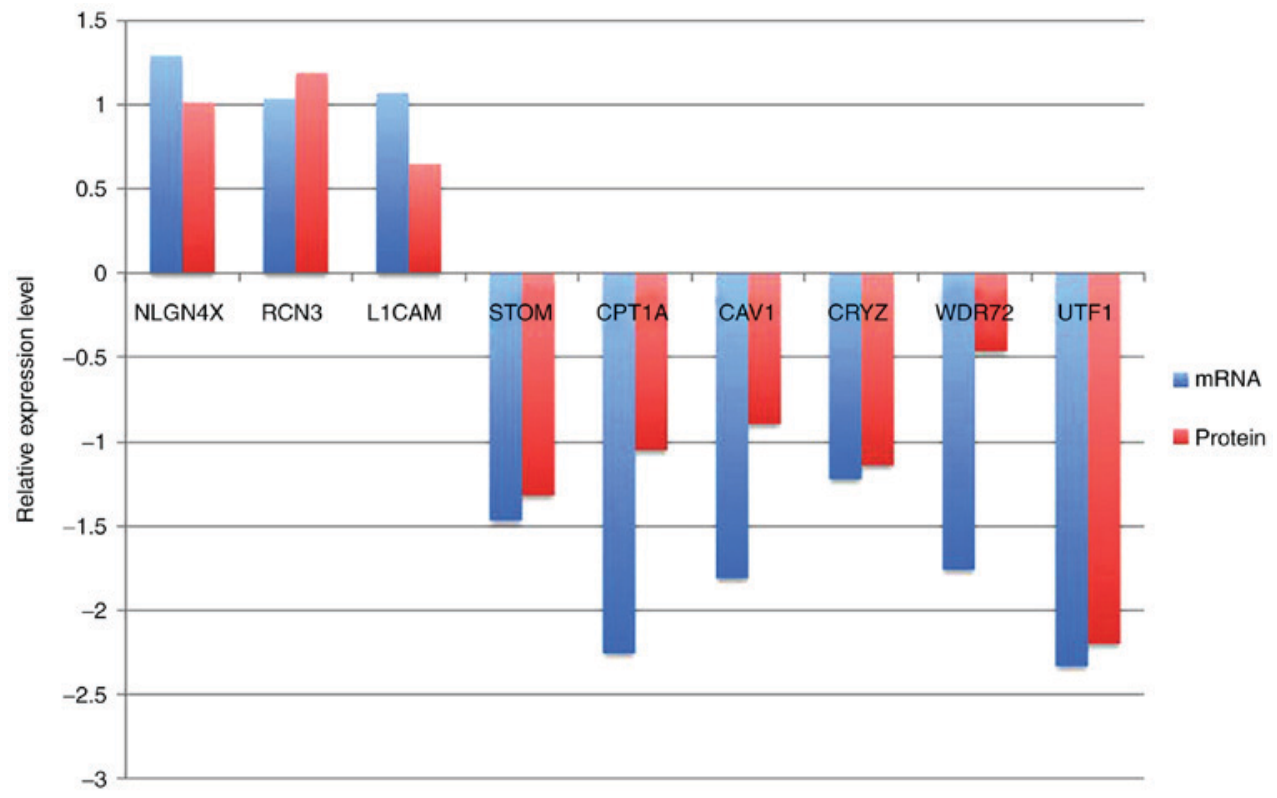

Figure 7. A total of nine proteins and their corresponding transcripts, identified to have a consistent expression pattern. The X-axis indicates transcripts and proteins. The Y-axis indicates upregulation or downregulation of expression. CAV1, caveolin 1; CPT1A, carnitine palmitoyltransferase 1A; CRYZ, crystallin- $\zeta$; L1CAM, L1 cell adhesion molecule; NLGN4X, neuroligin 4 X-linked; RCN3, reticulocalbin 3; STOM, stomatin; UTF1, undifferentiated embryonic cell transcription factor 1; WDR72, WD repeat domain 72.

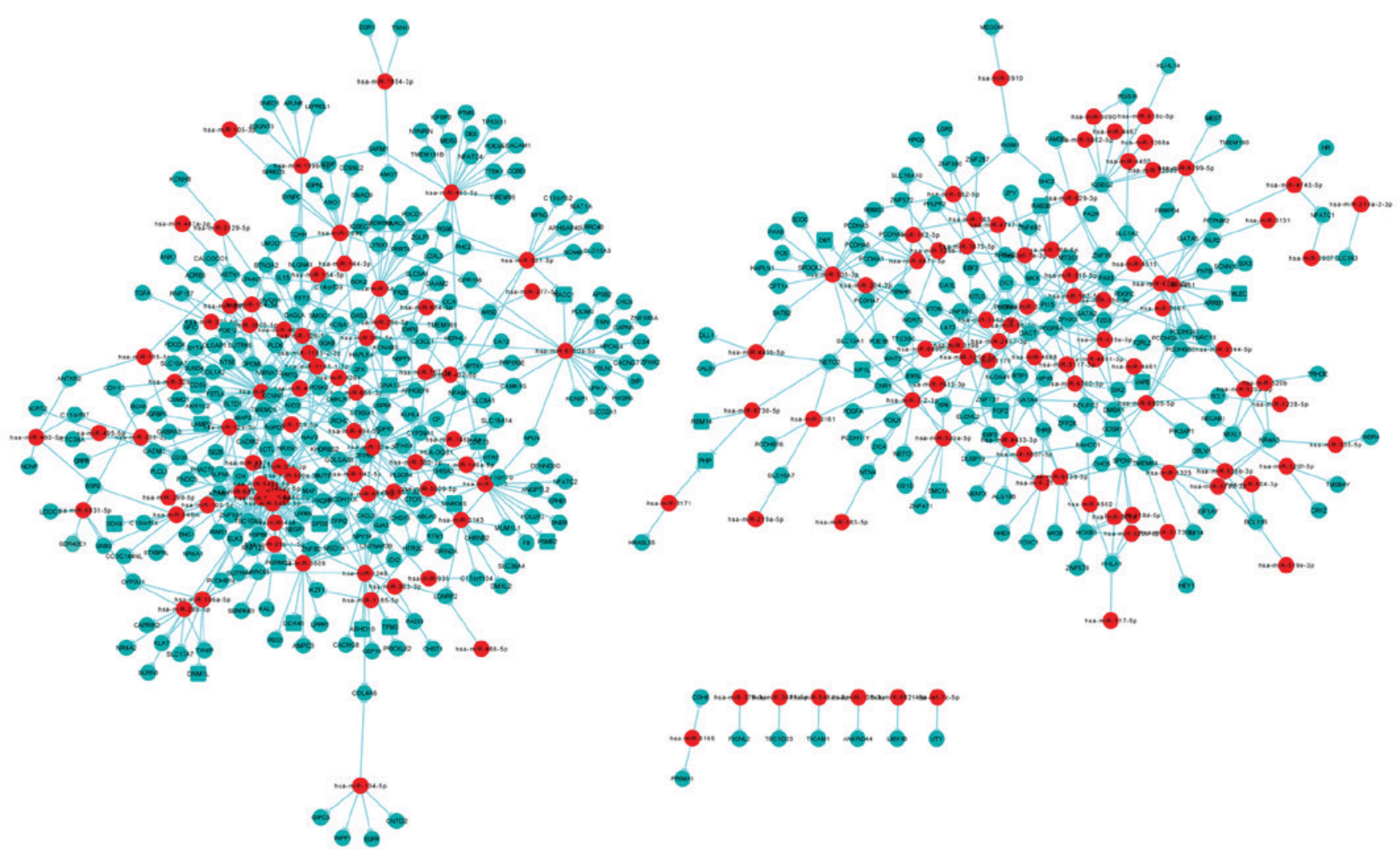

Figure 8. miRNA-protein-transcript regulatory network. The regulatory network comprises of 156 miRNAs, 26 proteins and 381 transcripts. The red circles indicate upregulated miRNAs, the blue circles represent downregulated transcripts and the blue squares indicate downregulated proteins. The lines signify coherent miRNA-transcript-protein-associated pairs. miRNA, microRNA.

cell binding in MF were identified as common GO terms for miRNAs and their target transcripts.

$\mathrm{AS}$ is an inherited disease caused by gene mutations that result in absence of the collagen IV network from basement membranes (9). The glomerular basement membrane (GBM) is the extracellular matrix component of the glomerular filtration barrier that lies between podocytes and endothelial cells. Pathological alterations in the GBM are specific characteristics 
of AS $(5,9)$. Notably, the results demonstrated that the miRNAs and their target transcripts were enriched in the 'membrane part' CC category. Studies have also revealed that podocyte dysfunction and the development of fibrosis are pathological alterations characteristic of AS $(2,48)$. Podocytes may bind to various immune deposits, thereby contributing to the development of fibrosis (49). This is consistent with the present results, which identified that the miRNAs and their target transcripts were enriched in 'binding' in the MF category. In the GO analysis of the miRNAs and their target proteins, 'binding' was also a significant term in the MF category.

The common KEGG pathways for the miRNAs and proteins were those involving carbon metabolism and cell adhesion molecules. Research has demonstrated that in addition to the inherited AS factors, acquired external factors serve an important role in the progression of the disease $(50,51)$. Metabolic abnormalities are an example of these acquired external factors $(52,53)$. During the development of kidney failure, the majority of patients with AS suffer from metabolic abnormalities, including high blood pressure, hyperlipidemia and high proteinuria $(54,55)$. Metabolic abnormalities contribute to the progression of kidney disease and subsequent kidney failure (56); however, the correction of metabolic disorders may considerably delay renal replacement therapy and accelerate the onset of end-stage kidney disease (57). Cell adhesion molecules are a principal mechanism of cell-cell and cell-matrix interactions. The growth, differentiation and organization of cells require cell-cell interactions (58). A previous study revealed that cadherin, a cell adhesion molecule, serves an important role in animal morphogenesis (59). Another study revealed that neural cell adhesion molecules were already present on the cells of the uninduced nephrogenic mesenchyme at the onset of kidney development (60). Therefore, it was hypothesized that morphological alterations occur in AS during the developmental process due to abnormalities in cell adhesion molecules, which in turn results in a thin and fractured GBM.

Cellular reproduction is controlled by protein-coding genes and noncoding regions, including those encoding for sRNAs. The expression of coding and noncoding genes may be markedly influenced by the structural features of the corresponding sRNA transcripts (61). miRNAs, a diverse class of highly conserved sRNAs, serve a critical role in fundamental biological processes, including cellular differentiation, apoptosis, cell proliferation and development $(62,63)$. The comprehensive characterization of miRNAs and transcripts in cell lines provides insight into the miRNA regulation of transcription, and enables the miRNA regulation of the corresponding target genes to be evaluated (64). In the present study, the regulatory network of miRNA-target transcripts revealed that they were mutually cross-regulated. Similarly, miRNA-protein interaction networks have long been studied in detail in various diseases. Numerous diseases have been linked to the misregulation or malfunction of proteins that interact with RNA (65). Thus, deciphering RNA-protein interactions at the molecular and cellular level is essential for understanding human physiology and disease (66). RNA-protein interactions are critical for the regulation of gene expression. Research over the last decade has demonstrated that RNA is invariably bound and frequently altered by proteins in cells, and that in biological environments, RNAs generally function together with proteins as RNA-protein complexes $(67,68)$. In the present study, it was identified that certain miRNAs and proteins were closely associated. An in-depth study of this interaction network may aid in understanding the pathogenesis of AS.

The aim of the present study was to identify potential important targets through regulatory network analysis of miRNAs, transcripts and proteins. Notably, hsa-miR-4775 had the greatest number of associations with proteins and target transcripts. In addition, hsa-miR-4775 was upregulated in AS-iPSCs, which was also verified using RT-qPCR. Recently a study revealed that hsa-miR-4775 promotes colorectal cancer invasion and metastasis (69). It was also reported that hsa-miR-4775 binds to the mRNAs of genes involved in the core apoptosis pathway, resulting in alterations which may lead to cancer, nephropathy and other diseases $(70,71)$. The EGFR family is a central element for cellular signal transduction and diversification (72). Upregulated EGFR was observed to be the most relevant transcript in the interaction analysis in the present study. EGFR is known to have a key role in chronic renal failure (73). Transgenic mice harboring the negative form of EGFR are resistant to the progression of renal lesions (74). Dawson et al (75) revealed that $E G F R$ was overexpressed in $75-90 \%$ of renal cell carcinomas and served an important role in tumor suppression or progression. Downregulated ELAVL1 was the most prominent protein in the network in the present study. This protein is a member of the ELAV family of RNA binding proteins that contain a number of RNA recognition motifs. ELAVL1 has been identified as a key post-transcriptional regulator and is implicated in cancer progression, particularly human renal cell carcinoma. ELAVL1 knockdown mice exhibited $60 \%$ inhibition of cell growth through inhibition of cell proliferation in addition to induction of cellular apoptosis (76). Therefore, it may be hypothesized that upregulated $E G F R$ was involved in chronic renal failure of AS. In addition, it was theorized that there was an association between EGFR and hsa-miR-4775 expression, which may have accelerated the core apoptosis of GBM cells. However, further studies are required to confirm this hypothesis.

In conclusion, 155 differentially expressed miRNAs, 1,168 differentially expressed transcripts and 383 differentially abundant proteins were identified in AS-iPSCs compared with NC-iPSCs. Using computational and systems techniques, integrated analysis of miRNA, transcript and protein expression data was performed. In addition, regulatory network maps were constructed to determine miRNA interactions with transcripts and proteins. To investigate the influence of miRNAs on biological processes, potential targets were predicted using miRanda, TargetScan and Pictar. The results demonstrated that hsa-miR-4775, ELAVL1 and EGFR were the most prominent miRNA, protein and transcript, respectively. RT-qPCR confirmed the upregulation of hsa-miR-4775 and EGFR. Target transcripts, proteins and miRNAs were used for GO enrichment analysis. 'Cellular process' in BP, 'membrane part' in CC and 'cell binding' in MF were common GO terms for miRNAs and target transcripts. 'Cell binding' in MF was also a common GO term for miRNAs and target proteins.

GenMAPP identified common KEGG pathways of miRNAs and proteins, which included 'carbon metabolism' and 'cell adhesion molecules'. Integration of multiple profiling datasets provides a novel way of examining gene regulation 
by miRNAs in conjunction with proteins and transcripts. This approach may enhance the understanding of the pathogenesis of AS and provide novel diagnostic and treatment strategies for AS.

\section{Acknowledgements}

The authors would like to acknowledge the Department of Collaborative Innovation Center for Diagnosis and Treatment of Infectious Diseases, Zhejiang University (Hangzhou, China) for assistance with the bioinformatics analysis.

\section{Funding}

The present study was supported by the Natural Science Foundation of China (grant no. 81671596) and Guangdong Province Chinese Scientific Project (grant no. 20170209).

\section{Availability of data and materials}

The miRNA sequences were submitted to NCBI and assigned the accession number SRP041435. The raw transcript sequence data has been deposited at NCBI under the accession number SRP041474.

\section{Authors' contributions}

HD and YD contributed to the conception and design of the research; WC wrote manuscript and supervised the report; WC and DT collected information, assembly the tables and drawn the figures; DT performed the research experiments and interpreted of data; WC critically reviewed.

\section{Ethics approval and consent to participate}

The study protocol and consent forms were approved by the Ethics Committee of Jinan University and adhered to the guidelines set forth in the Declaration of Helsinki.

\section{Patient consent for publication}

Healthy participants and patients with AS provided written informed consent.

\section{Competing interests}

The authors declare that they have no competing interests.

\section{References}

1. Heidet L and Gubler MC: The renal lesions of Alport syndrome. J Am Soc Nephrol 20: 1210-1215, 2009.

2. Kruegel J, Rubel D and Gross O: Alport syndrome-insights from basic and clinical research. Nat Rev Nephrol 9: 170-178, 2013.

3. Endreffy E, Ondrik Z, Ivanyi B, Maróti Z, Bereczki C, Haszon I, Györke Z, Worum E, Németh K, Rikker C, et al: Collagen type IV nephropathy: Genetic heterogeneity examinations in affected hungarian families. Mol Cell Probes 25: 28-34, 2011.

4. Thorner PS: Alport syndrome and thin basement membrane nephropathy. Nephron Clin Pract 106: c82-c88, 2007.

5. Haas M: Alport syndrome and thin glomerular basement membrane nephropathy: A practical approach to diagnosis. Arch Pathol Lab Med 133: 224-232, 2009.
6. Miao Y, Xiong J, Zhang X, Huang H, Yu L, Chen J, Deng W, $\mathrm{Xu} \mathrm{H}$, Liu R, Xiang C, et al: Genetic diagnosis of polycystic kidney disease, Alport syndrome, and thalassemia minor in a large Chinese family. Clin Sci (Lond) 131: 2427-2438, 2017.

7. Weber S, Strasser K, Rath S, Kittke A, Beicht S, Alberer M, Lange-Sperandio B, Hoyer PF, Benz MR, Ponsel S, et al: Identification of 47 novel mutations in patients with alport syndrome and thin basement membrane nephropathy. Pediatr Nephrol 31: 941-955, 2016.

8. Nozu K, Vorechovsky I, Kaito H, Fu XJ, Nakanishi K, Hashimura Y, Hashimoto F, Kamei K, Ito S, Kaku Y, et al: X-linked Alport syndrome caused by splicing mutations in COL4A5. Clin J Am Soc Nephrol 9: 1958-1964, 2014.

9. Lin X, Suh JH, Go G and Miner JH: Feasibility of repairing glomerular basement membrane defects in alport syndrome. J Am Soc Nephrol 25: 687-692, 2014.

10. Guo Y, Yuan J, Liang H, Xiao J, Xu H, Yuan L, Gao K, Wu B, Tang Y, Li X and Deng H: Identification of a novel COL4A5 mutation in a Chinese family with X-linked Alport syndrome using exome sequencing. Mol Biol Rep 41: 3631-3635, 2014.

11. Kim VN and Nam JW: Genomics of microRNA. Trends Genet 22: 165-173, 2006.

12. Ghosh Z, Chakrabarti J and Mallick B: miRNomics-the bioinformatics of microRNA genes. Biochem Biophys Res Commun 363: 6-11, 2007.

13. Chung AC, Yu X and Lan HY: MicroRNA and nephropathy: Emerging concepts. Int J Nephrol Renovasc Dis 6: 169-179, 2013.

14. Chen W, Lin X, Huang J, Tan K, Chen Y, Peng W, Li W and Dai Y: Integrated profiling of microRNA expression in membranous nephropathy using high-throughput sequencing technology. Int J Mol Med 33: 25-34, 2014

15. Tan K, Chen J, Li W, Chen Y, Sui W, Zhang Y and Dai Y: Genome-wide analysis of microRNAs expression profiling in patients with primary IgA nephropathy. Genome 56: 161-169, 2013.

16. Dai Y, Huang YS, Tang M, Lv TY, Hu CX, Tan YH, Xu ZM and Yin YB: Microarray analysis of microRNA expression in peripheral blood cells of systemic lupus erythematosus patients. Lupus 16: 939-946, 2007.

17. Hafner M, Landthaler M, Burger L, Khorshid M, Hausser J, Berninger P, Rothballer A, Ascano M Jr, Jungkamp AC, Munschauer $\mathrm{M}$, et al: Transcriptome-wide identification of RNA-binding protein and microRNA target sites by PAR-CLIP. Cell 141: 129-141, 2010.

18. Woroniecka KI, Park AS, Mohtat D, Thomas DB, Pullman JM and Susztak K: Transcriptome analysis of human diabetic kidney disease. Diabetes 60: 2354-2369, 2011.

19. Famulski KS, Broderick G, Einecke G, Hay K, Cruz J, Sis B, Mengel $\mathrm{M}$ and Halloran PF: Transcriptome analysis reveals heterogeneity in the injury response of kidney transplants. Am J Transplant 7: 2483-2495, 2007.

20. Kapoor I, Pal P, Lochab S, Kanaujiya JK and Trivedi AK: Proteomics approaches for myeloid leukemia drug discovery. Expert Opin Drug Discov 7: 1165-1175, 2012.

21. Raimondo F, Corbetta S, Morosi L, Chinello C, Gianazza E, Castoldi G, Di Gioia C, Bombardi C, Stella A, Battaglia C, et al: Urinary exosomes and diabetic nephropathy: A proteomic approach. Mol Biosyst 9: 1139-1146, 2013.

22. Park MR, Wang EH, Jin DC, Cha JH, Lee KH, Yang CW, Kang CS and Choi YJ: Establishment of a 2-D human urinary proteomic map in IgA nephropathy. Proteomics 6: 1066-1076, 2006.

23. Ebert AD, Liang P and Wu JC: Induced pluripotent stem cells as a disease modeling and drug screening platform. J Cardiovasc Pharmacol 60: 408-416, 2012.

24. Hirschi KK, Li S and Roy K: Induced pluripotent stem cells for regenerative medicine. Annu Rev Biomed Eng 16: 277-294, 2014.

25. Qian K, Huang CT, Chen H, Blackbourn LW IV, Chen Y, Cao J, Yao L, Sauvey C, Du Z and Zhang SC: A simple and efficient system for regulating gene expression in human pluripotent stem cells and derivatives. Stem Cells 32: 1230-1238, 2014.

26. Hao J, Duan FF and Wang Y: MicroRNAs and RNA binding protein regulators of microRNAs in the control of pluripotency and reprogramming. Curr Opin Genet Dev 46: 95-103, 2017.

27. Mallanna SK and Rizzino A: Emerging roles of microRNAs in the control of embryonic stem cells and the generation of induced pluripotent stem cells. Dev Biol 344: 16-25, 2010.

28. Chen W, Huang J, Yu X, Lin X and Dai Y: Generation of induced pluripotent stem cells from renal tubular cells of a patient with Alport syndrome. Int J Nephrol Renovasc Dis 8: 101-109, 2015. 
29. Audic S and Claverie JM: The significance of digital gene expression profiles. Genome Res 7: 986-995, 1997.

30. Livak KJ and Schmittgen TD: Analysis of relative gene expression data using real-time quantitative PCR and the 2(-Delta Delta C(T)) method. Methods 25: 402-408, 2001.

31. Van Roey K and Davey NE: Motif co-regulation and co-operativity are common mechanisms in transcriptional, post-transcriptiona and post-translational regulation. Cell Commun Signal 13: 45, 2015.

32. Singh VK, Kalsan M, Kumar N, Saini A and Chandra R: Induced pluripotent stem cells: Applications in regenerative medicine, disease modeling, and drug discovery. Front Cell Dev Biol 3: 2, 2015.

33. Kondo Y, Toyoda T, Inagaki N and Osafune K: iPSC technologybased regenerative therapy for diabetes. J Diabetes Investig 9 : 234-243, 2018.

34. Yoshida Y and Yamanaka S: Induced pluripotent stem cells 10 years later: For cardiac applications. Circ Res 120: 1958-1968, 2017.

35. Jung verdorben J, Till A and Brustle O: Induced pluripotent stem cell-based modeling of neurodegenerative diseases: A focus on autophagy. J Mol Med (Berl) 95: 705-718, 2017.

36. Jang $Y Y$ and $Y e ~ Z$ : Gene correction in patient-specific iPSCs for therapy development and disease modeling. Hum Genet 135 1041-1058, 2016.

37. Zou J, Mali P,Huang X, Dowey SN and Cheng L: Site-specific gene correction of a point mutation in human iPS cells derived from an adult patient with sickle cell disease. Blood 118: 4599-4608, 2011

38. Yusa K, Rashid ST, Strick-Marchand H, Varela I, Liu PQ Paschon DE, Miranda E, Ordóñez A, Hannan NR, Rouhani FJ, et al: Targeted gene correction of alpha1-antitrypsin deficiency in induced pluripotent stem cells. Nature 478: 391-394, 2011.

39. Garate Z, Quintana-Bustamante O, Crane AM, Olivier E, Poirot L, Galetto R, Kosinski P, Hill C, Kung C, Agirre X, et al: Generation of a high number of healthy erythroid cells from gene-edited pyruvate kinase deficiency patient-specific induced pluripotent stem cells. Stem Cell Reports 5: 1053-1066, 2015.

40. Brix J, Zhou Y and Luo Y: The epigenetic reprogramming roadmap in generation of iPSCs from somatic cells. J Genet Genomics 42: 661-670, 2015 .

41. Liang $\mathrm{G}$ and Zhang Y: Embryonic stem cell and induced pluripotent stem cell: An epigenetic perspective. Cell Res 23: 49-69, 2013

42. Li N, Long B, Han W, Yuan S and Wang K: microRNAs: Important regulators of stem cells. Stem Cell Res Ther 8: 110, 2017.

43. Wang T, Shi SB and Sha HY: MicroRNAs in regulation of pluripotency and somatic cell reprogramming: Small molecule with big impact. RNA Biol 10: 1255-1261, 2013.

44. Zhang H, Xue C, Shah R, Bermingham K, Hinkle CC, Li W, Rodrigues A, Tabita-Martinez J, Millar JS, Cuchel M, et al: Functional analysis and transcriptomic profiling of iPSC-derived macrophages and their application in modeling mendelian disease. Circ Res 117: 17-28, 2015

45. Huang X, Wang Y, Yan W, Smith C, Ye Z, Wang J, Gao Y, Mendelsohn L and Cheng L: Production of gene-corrected adult beta globin protein in human erythrocytes differentiated from patient iPSCs after genome editing of the sickle point mutation. Stem Cells 33: 1470-1479, 2015.

46. Chen F, Zhang G, Yu L, Feng Y, Li X, Zhang Z, Wang Y, Sun D and Pradhan S: High-efficiency generation of induced pluripotent mesenchymal stem cells from human dermal fibroblasts using recombinant proteins. Stem Cell Res Ther 7: 99, 2016.

47. Poos K, Smida J, Nathrath M, Maugg D, Baumhoer D and Korsching E: How microRNA and transcription factor co-regulatory networks affect osteosarcoma cell proliferation. PLoS Comput Biol 9: e1003210, 2013.

48. Nagata M: Podocyte injury and its consequences. Kidney Int 89: 1221-1230, 2016

49. Wang H, Yue Z, Wu J, Liu T, Mo Y, Jiang X and Sun L: The accumulation of VEGFA in the glomerular basement membrane and its relationship with podocyte injury and proteinuria in alport syndrome. PLoS One 10: e0135648, 2015.

50. Hudson BG, Tryggvason K, Sundaramoorthy M and Neilson EG: Alport's syndrome, goodpasture's syndrome, and type IV collagen. N Engl J Med 348: 2543-2556, 2003.

51. Kluth DC and Rees AJ: Anti-glomerular basement membrane disease. J Am Soc Nephrol 10: 2446-2453, 1999.

52. Chen J, Gu D, Chen CS, Wu X, Hamm LL, Muntner P, Batuman V, Lee CH, Whelton PK and He J: Association between the metabolic syndrome and chronic kidney disease in chinese adults. Nephrol Dial Transplant 22: 1100-1106, 2007.

53. Peralta CA, Kurella M, Lo JC and Chertow GM: The metabolic syndrome and chronic kidney disease. Curr Opin Nephrol Hypertens 15: 361-365, 2006
54. Jarad G, Knutsen RH, Mecham RP and Miner JH: Albumin contributes to kidney disease progression in Alport syndrome. Am J Physiol Renal Physiol 311: F120-F130, 2016.

55. Gross O,Licht C,Anders HJ,Hoppe B,BeckB, Tönshoff B,Höcker B, Wygoda S, Ehrich JH, Pape L, et al: Early angiotensin-converting enzyme inhibition in Alport syndrome delays renal failure and improves life expectancy. Kidney Int 81: 494-501, 2012.

56. Kurella M, Lo JC and Chertow GM: Metabolic syndrome and the risk for chronic kidney disease among nondiabetic adults. J Am Soc Nephrol 16: 2134-2140, 2005.

57. Klahr S, Levey AS, Beck GJ, Caggiula AW, Hunsicker L, Kusek JW and Striker G: The effects of dietary protein restriction and blood-pressure control on the progression of chronic renal disease. Modification of diet in renal disease study group N Engl J Med 330: 877-884, 1994

58. Gumbiner BM: Cell adhesion: The molecular basis of tissue architecture and morphogenesis. Cell 84: 345-357, 1996.

59. Halbleib JM and Nelson WJ: Cadherins in development: Cell adhesion, sorting, and tissue morphogenesis. Genes Dev 20: 3199-3214, 2006

60. Klein G, Langegger M, Goridis C and Ekblom P: Neural cell adhesion molecules during embryonic induction and development of the kidney. Development 102: 749-761, 1988.

61. Lagos-Quintana M, Rauhut R, Lendeckel W and Tuschl T: Identification of novel genes coding for small expressed RNAs. Science 294: 853-858, 2001

62. Ambros V: The functions of animal microRNAs. Nature 431: 350-355, 2004

63. Cui Q, Yu Z, Pan Y, Purisima EO and Wang E: MicroRNAs preferentially target the genes with high transcriptional regulation complexity. Biochem Biophys Res Commun 352: 733-738, 2007.

64. Glisovic T, Bachorik JL, Yong J and Dreyfuss G: RNA-binding proteins and post-transcriptional gene regulation. FEBS Lett 582: 1977-1986, 2008.

65. Li JH, Liu S, Zhou H, Qu LH and Yang JH: starBase v2.0 Decoding miRNA-ceRNA, miRNA-ncRNA and protein-RNA interaction networks from large-scale CLIP-Seq data. Nucleic Acids Res 42: D92-D97, 2014.

66. Jankowsky E and Harris ME: Specificity and nonspecificity in RNA-protein interactions. Nat Rev Mol Cell Biol 16: 533-544, 2015.

67. Buenrostro JD, Araya CL, Chircus LM, Layton CJ, Chang HY, Snyder MP and Greenleaf WJ: Quantitative analysis of RNA-protein interactions on a massively parallel array reveals biophysical and evolutionary landscapes. Nat Biotechnol 32: 562-568, 2014

68. Hennig J and Sattler M: Deciphering the protein-RNA recognition code: Combining large-scale quantitative methods with structural biology. Bioessays 37: 899-908, 2015.

69. Zhao S, Sun H, Jiang W, Mi Y, Zhang D, Wen Y, Cheng D, Tang H, Wu S, Yu Y, et al: miR-4775 promotes colorectal cancer invasion and metastasis via the Smad7/TGF $\beta$-mediated epithelial to mesenchymal transition. Mol Cancer 16: 12, 2017.

70. Lima RT, Busacca S, Almeida GM, Gaudino G, Fennell DA and Vasconcelos MH: MicroRNA regulation of core apoptosis pathways in cancer. Eur J Cancer 47: 163-174, 2011.

71. Ouyang L, Shi Z, Zhao S, Wang FT, Zhou TT, Liu B and Bao JK: Programmed cell death pathways in cancer: A review of apoptosis, autophagy and programmed necrosis. Cell Prolif 45: 487-498, 2012.

72. Prenzel N, Fischer OM, Streit S, Hart S and Ullrich A: The epidermal growth factor receptor family as a central element for cellular signal transduction and diversification. Endocr Relat Cancer 8: 11-31, 2001

73. Lautrette A, Li S, Alili R, Sunnarborg SW, Burtin M, Lee DC, Friedlander G and Terzi F: Angiotensin II and EGF receptor cross-talk in chronic kidney diseases: A new therapeutic approach. Nat Med 11: 867-874, 2005.

74. Friedlander G and Terzi F: Angiotensin and EGF receptor cross-talk in chronic kidney diseases: Towards a new therapeutic approach. Bull Acad Natl Med 190: 927-934, 2006 (In French).

75. Dawson NA, Guo C, Zak R, Dorsey B, Smoot J, Wong J and Hussain A: A phase II trial of gefitinib (Iressa, ZD1839) in stage IV and recurrent renal cell carcinoma. Clin Cancer Res 10: 7812-7819, 2004.

76. Danilin S, Sourbier C, Thomas L, Lindner V, Rothhut S, Dormoy V, Helwig JJ, Jacqmin D, Lang $\mathrm{H}$ and Massfelder T: Role of the RNA-binding protein HuR in human renal cell carcinoma. Carcinogenesis 31: 1018-1026, 2010.

This work is licensed under a Creative Commons Attribution-NonCommercial-NoDerivatives 4.0 International (CC BY-NC-ND 4.0) License. 